\title{
Central alarin ameliorated insulin resistance of adipocytes in type 2 diabetic rats
}

\author{
Lili Guo ${ }^{4}$, Penghua Fang ${ }^{3}$, Mei $\mathrm{Yu}^{3}$, Mingyi Shi ${ }^{2}$, Ping Bo ${ }^{1,2}$ and Zhenwen Zhang ${ }^{1}$ \\ ${ }^{1}$ Department of Endocrinology, Clinical Medical College, Yangzhou University, Nantong West Street No. 98, \\ Yangzhou, Jiangsu 225001, China \\ ${ }^{2}$ Medical College, Research Institute of Combined Chinese Traditional and Western Medicine, Yangzhou University, \\ Yangzhou, Jiangsu 225001, China \\ ${ }^{3}$ Department of Physiology, Hanlin College, Nanjing University of Chinese Medicine, Taizhou, Jiangsu 225300, China \\ ${ }^{4}$ Department of Physical Education, Chuzhou College, Chuzhou, Anhui Province 239012, China
}

\author{
Correspondence \\ should be addressed \\ to Z Zhang or P Bo \\ Emails \\ shimyyz@126.com or \\ yzzzw@medmail.com.cn
}

\begin{abstract}
Alarin, a regulatory peptide, belongs to the galanin family and plays the same regulatory roles as galanin in orexigenic activity and energy metabolism. Our previous studies had found that galanin might facilitate insulin sensitivity via activation of its central receptors. To date, little is known about whether central alarin may exert similar effects on insulin sensitivity. In order to investigate this, alarin and its specific antagonist, alarin 6-25Cys, were administered into the cerebral ventricles of type 2 diabetic rats (T2DR) to evaluate the changes in insulin resistance. The results indicated that central treatment with alarin significantly increased the body weight of animals, the 2-( $\mathrm{N}$-(7-nitrobenz-2-oxa-1,3-diazol4-yl)amino)-2-deoxyglucose uptake, the plasma adiponectin levels, the glucose infusion rates in hyperinsulinemic-euglycemic clamp tests, the vesicle-associated membrane protein 2 as well as glucose transporter 4 (GLUT4 (SLC2A4)) protein and mRNA levels, and the ratios of GLUT4 contents in plasma membranes to total cell membranes in adipocytes, but reduced blood glucose and plasma retinol-binding protein 4 levels. These effects of alarin may be inhibited by pretreatment with alarin $6-25$ Cys. The above-mentioned results suggest that the central alarin projective system may facilitate insulin sensitivity and glucose uptake via the increase in GLUT4 content and GLUT4 translocation from intracellular pools to plasma membranes in T2DR.
\end{abstract}

\author{
Key Words \\ - GLUT4 \\ - adipocytes \\ - alarin \\ - brain \\ - insulin resistance
}

\section{Introduction}

Alarin, isolated first in 2006 from the gangliocytes of human neuroblastic tumors, is a 25 amino acid neuropeptide (Santic et al. 2006). This peptide is a member of the galanin peptide family that includes galanin and galanin-like peptide (GALP), etc. (Santic et al. 2007, Van Der Kolk et al. 2010). Alarin shares the same first five amino acids as GALP in the N-terminal end, encoding a splice variant of the Galp gene in exclusion of exon 3, but the following 20 amino acids show no sequence identity to galanin or GALP (Santic et al. 2006, Wada et al. 2013). Sequence identity of this peptide between macaque and human is $96 \%$, and that between rat and murine is $88 \%$ (Santic et al. 2006, Fraley et al. 2013). In the CNS, alarinlike immunoreactivity was observed in a variety of areas throughout the entire brain, mainly distributed in the locus coeruleus, the locus subcoeruleus of midbrains and 
hindbrains in rats, the medial preoptic area, the accessory olfactory bulb, amygdala, and hypothalamus in mice (Eberhard et al. 2012).

A critical step to effect whole-body glucose homeostasis is insulin-stimulated glucose uptake, which needs the help of glucose transporters. Of the glucose transporter family, the most important and abundant member is glucose transporter 4 (GLUT4; Absalan et al. 2012). After GLUT4 vesicles are trafficked from an intracellular pool to dock and fuse with the plasma membrane of cells, GLUT4 can benefit glucose uptake from extracellular environment into adipocytes and myocytes (Liang et al. 2012). Insulin receptor-mediated signaling cascades trigger dramatic acceleration of GLUT4 exocytosis and inhibition of its endocytosis to recruit GLUT4 onto the cell surface, resulting in an increase in glucose flux into the cells. The defective translocation of GLUT4 to the cell surface in response to elevated circulating insulin levels results in insulin resistance (Song et al. 2013).

Similar to galanin and GALP, alarin stimulates an orexigenic behavior as well as increasing body weight and secretion of luteinizing hormone and gonadotropinreleasing hormone in murine models (Boughton et al. 2010, Van Der Kolk et al. 2010, Fraley et al. 2013). Recently, we and others have found that central galanin may facilitate GLUT4 translocation and insulin sensitivity in healthy and type 2 diabetic rats (T2DR) (Guo et al. 2012, Liang et al. 2012, Zhang et al. 2012, Bu et al. 2013). However, little is known as to whether central alarin is involved in a similar role of ameliorating insulin resistance. In this study, we investigated this action of alarin in adipocytes via i.c.v. administration of alarin and alarin antagonist, alarin 6-25Cys (ala6-25Cys) (Fraley et al. 2013), in T2DR.

\section{Materials and methods}

\section{Materials}

Alarin was purchased from the Peptide Institute (SaitoAsagi, Ibaraki-shi, Osaka, Japan) and 2-(N-(7-nitrobenz-2oxa-1,3-diazol-4-yl)amino)-2-deoxy-D-glucose (2-NBDG) and Trizol reagent were purchased from Invitrogen (Burlington, Ontario, Canada). Streptozotocin was obtained from Sigma-Aldrich, Inc. GLUT4 and vesicleassociated membrane protein 2 (VAMP2) antibodies were obtained from Santa Cruz Biotechnology (Santa Cruz, CA, USA). Retinol-binding protein 4 (RBP4) and adiponectin ELISA kits were obtained from Uscn Life Science (Wuhan, China). Ala6-25Cys (SSPFPPRPTRAGRETQLLRSC) were custom synthesized by GL Biochem (Shanghai, China).

\section{Animals and i.c.v. injection}

All animal procedures used in this study were performed in accordance with the Guiding Principles for Care and Use of Experimental Animals. The experiments carried out in this study were approved by the Animal Studies Committee of Yangzhou University. The 4-week-old male Wistar rats purchased from Yangzhou University Animal Center were housed in cages in groups of five under a controlled temperature $\left(22^{\circ} \mathrm{C}\right)$ and photoperiod cycle of $12 \mathrm{~h}$ light: $12 \mathrm{~h}$ darkness. Rats were fed on a high-fat diet (59\% fat, $21 \%$ protein, and $20 \%$ carbohydrate) and tap water ad libitum for 8 weeks. Then, rats were intraperitoneally injected streptozotocin $(35 \mathrm{mg} / \mathrm{kg}$ i.p.) under a fasted state. On the 7th day after the injection, the animals with fasting glucose concentration over $11.1 \mathrm{mmol} / \mathrm{l}$ were taken as models of T2DR (Zhang et al. 2012).

The 64 diabetic rats were randomly distributed into four groups as follows: diabetic control $(n=16)$, diabetic group with alarin $(n=16)$, diabetic group with ala6-25Cys $(n=16)$, and diabetic group with ala6-25Cys + alarin $(n=16)$. In addition, 16 healthy rats formed the healthy control group.

Rats were anesthetized with 3\% amobarbital sodium $(50 \mathrm{mg} / \mathrm{kg}$ i.p.), and were then steadily set on a stereotaxic frame as described previously (Zhang et al. 2012). A 22-gauged stainless steel guide cannula was inserted into the lateral ventricle $(\mathrm{AP}),-0.8 \mathrm{~mm} ; \mathrm{L}, 1.4 \mathrm{~mm}$; and $\mathrm{V}$, $3.3 \mathrm{~mm}$. After recuperation for 7 days, rats were, respectively, injected $2.0 \mathrm{nmol}$ alarin, $5.0 \mathrm{nmol}$ ala6-25Cys, $2.0 \mathrm{nmol}$ alarin $+5.0 \mathrm{nmol}$ ala6-25Cys, or $5 \mu \mathrm{l}$ artificial cerebrospinal fluid (in mM: $133.3 \mathrm{NaCl}, 0.6 \mathrm{NaH}_{2} \mathrm{PO}_{4}, 1.2$ $\mathrm{MgCl}_{2}, 1.3 \mathrm{CaCl}_{2}, 3.4 \mathrm{KCl}, 32.0 \mathrm{NaHCO}_{3}$, and 3.4 glucose, pH 7.4 by $0.5 \mathrm{M} \mathrm{HCl}$ ) through the guide cannula once a day for 7 days.

\section{Hyperinsulinemic-euglycemic clamp}

Fasted for $12 \mathrm{~h}$ after the last i.c.v. injection, half of rats in every group $(n=8)$ were anesthetized as described above and subjected to a hyperinsulinemic-euglycemic clamp test as described previously (Guo et al. 2012). A primed continuous infusion of human regular insulin was initiated at a rate of $2 \mathrm{mU} / \mathrm{kg}$ per min through a jugular vein catheter until the experimental end. Glucose solution (20\%) was infused at variable rates as appropriate to clamp euglycemia at $5 \mathrm{mmol} / \mathrm{l}$ level. The whole-body glucose turnover was determined at 5-min intervals. The glucose infusion rate was adjusted corresponding to the last six samplings at the stable clamp level.

Published by Bioscientifica Ltd 


\section{2-NBDG test and collection of samples}

To enhance 2-NBDG uptake, the remaining rats in each group $(n=8)$ were fasted for $24 \mathrm{~h}$ after the last i.c.v. injection (Sheth et al. 2009), and then intravenously injected $1 \mathrm{mg} / \mathrm{kg}$ 2-NBDG. At 30 min after drug injections, rats were killed by an overdose of nembutal $(200 \mathrm{mg} / \mathrm{kg}$ ). The epididymal fat pad and ventral fat of rats were collected, washed, minced, homogenized, and centrifuged at $1300 \mathrm{~g}$ for $10 \mathrm{~min}$ at $4{ }^{\circ} \mathrm{C}$. The supernatant was used to measure the 2-NBDG contents using a Multichannel Microplate Reader (Biotek, USA). The turbid liquid was frozen at $-80{ }^{\circ} \mathrm{C}$ until further analysis.

\section{Measurement of plasma RBP4 and adiponectin levels}

The RBP4 and adiponectin levels were quantified using competitive RBP4 and adiponectin ELISA kits respectively.

\section{Subcellular fractionation of adipocytes}

Membranes of adipocytes were separated as described previously (Zhang et al. 2012). Briefly, the turbid liquid was centrifuged at $31000 \boldsymbol{g}$ for $1 \mathrm{~h}$ at $4{ }^{\circ} \mathrm{C}$. The pellet from the spin was layered on 25 and $50 \%$ sucrose gradients and centrifuged at $75000 \boldsymbol{g}$ for $1 \mathrm{~h}$. The interphase was removed and spun at $39000 \boldsymbol{g}$ for $20 \mathrm{~min}$ to yield the plasma membranes.

\section{Western blot assay}

The GLUT4 levels in the adipocytes were analyzed using a western blot method described previously (Liang et al. 2012). After $50 \mu \mathrm{g}$ of samples were separated on a $12 \%$ polyacrylamide gel, the separated proteins were transferred onto a polyvinylidene difluoride filter membrane. The membranes were probed with GLUT4 or VAMP2 antibody solutions, followed by the secondary antibody at room temperature. The immunoreactive bands were visualized by chemiluminescence and quantified by densitometry using an HPIAS-2000 Image Analysis System (Championimages, China). $\beta$-actin was taken as an internal control. The sum of the GLUT4 concentration in plasma membranes and that in intracellular membranes was calculated as that of total cell membranes.

\section{Real-time PCR}

The total mRNA was isolated from $100 \mathrm{mg}$ frozen samples using Trizol. Isolated mRNA was quantified using a spectrometer at a wavelength of $260 \mathrm{~nm}$ and verified by formaldehyde agarose gel electrophoresis. cDNA was synthesized from $2 \mu \mathrm{g}$ total RNA using Moloney murine leukemia virus (MMLV) reverse transcriptase. Quantification of mRNAs was carried out using real-time fluorescent detection in an ExicyclerTM 96 PCR machine (LG Company, Korea) under the below conditions: $95^{\circ} \mathrm{C} \times 30 \mathrm{~min}, 40 \times$ $\left(95^{\circ} \mathrm{C} \times 30 \mathrm{~s}, 60^{\circ} \mathrm{C} \times 30 \mathrm{~s}, 72^{\circ} \mathrm{C} \times 60 \mathrm{~s}\right.$ ). The primers used were as follows: Glut4 (Slc2a4), forward 5'-AGGCAAGAGGGAGTTACCACT-3' and reverse 5'-GGTGGCCAAGGGGATG-3' and $\beta$-actin, forward $5^{\prime}$-GGCTGTGTTGTCCCTGTATG- $3^{\prime}$ and reverse $5^{\prime}$-AATGTCACGCACGATTTCC- $3^{\prime}$. The real-time PCR data were analyzed by the $2^{-\Delta \Delta C T}$ method with $\beta$-actin as an internal control, according to the method described previously (Zhang et al. 2012).

\section{Statistical analysis}

Statistical differences between means were evaluated by one-way ANOVA followed by Tukey's test. Parametric data were expressed as mean \pm s.E.M. with $P<0.05$ considered as significant.

\section{Results}

\section{The changes in blood glucose levels and body weight}

Compared with treatment with the vehicle, the i.c.v. injection of alarin significantly decreased the blood glucose levels $(F(4,40)=96.8, P<0.0001)$, but enhanced the body weight of the animals $(F(4,40)=17.1, P<0.0001)$. Compared with each diabetic control, the glycemic levels in rats treated with alarin were reduced by $46.1 \%(P<0.01)$ and body weight increased by $12.9 \%(P<0.05)$, but the former in rats treated with ala6-25Cys was enhanced by $12.8 \%(P<0.05)$ and the latter decreased by $5.6 \%(P>0.05)$ respectively (Fig. 1$)$. Administration of ala6-25Cys in various degrees reversed the alarininduced alleviation of hyperglycemia as well as gain of body weight in the animals. In the alarin +ala6-25Cys group, the blood glucose levels were increased by $28.7 \%(P<0.05)$, but body weight was decreased by $10.6 \%(P<0.05)$ compared with the alarin group, whereas the former was decreased by $38.5 \%(P<0.01)$, but the latter was increased by $6.9 \%$ $(P>0.05)$ when compared with the ala6-25Cys group. In the diabetic control group, the blood glucose concentration was elevated by $149.7 \%(P<0.01)$ and the body weight was reduced by $18.8 \%(P<0.01)$ compared with healthy controls.

\section{Plasma RBP4 and adiponectin concentration}

Adipose tissue, beyond the traditional concept as a passive energy reservoir, has been regarded as an active endocrine

Published by Bioscientifica Ltd. 


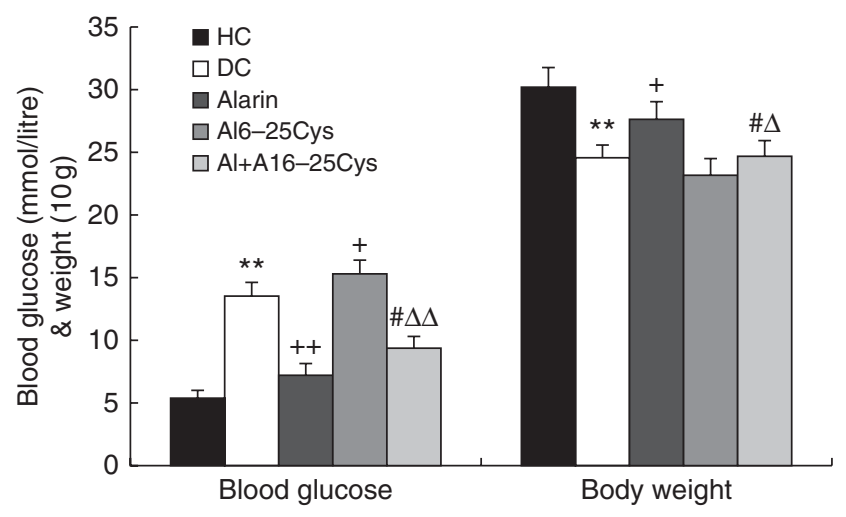

Figure 1

The changes in blood glucose levels and body weight after the central injection of alarin in type 2 diabetic rats $(n=8)$. The blood glucose levels were lower, but the body weight was higher in the alarin group compared with diabetic controls (DCs), which may be abolished by the alarin antagonist, alarin 6-25Cys. The former was higher, but the latter was lower in DCs compared with healthy controls (NCs). While the former was lower, the latter was higher in the alarin + alarin 6-25Cys group than in the alarin 6-25Cys group. Moreover, the glycemic levels were higher in the alarin 6-25Cys group compared with DCs. All data shown are the means \pm s.E.M. ${ }^{*} * P<0.01$ vs NCs; ${ }^{+} P<0.05,{ }^{++} P<0.01$ vs $D C s ;{ }^{\#} P<0.05$ vs alarin group; ${ }^{\Delta} P<0.05,{ }^{\Delta \Delta} P<0.01$ vs alarin $6-25$ Cys group.

organ to synthesize and secrete adipokines, such as RBP4 and adiponectin, which are linked to insulin resistance (Patel et al. 2012, Kotnik et al. 2013). As shown in Fig. 2, the central injection of alarin significantly reduced the RBP4 contents $(F(4,40)=16.25, P<0.0001)$, but elevated the adiponectin contents $(F(4,40)=26.18, P<0.0001)$. Compared with the diabetic controls, the RBP4 concentration was decreased by $15.8 \%(P<0.05)$ and the adiponectin contents were elevated by $31.7 \%(P<0.01)$ in the alarin group, while the former was increased by $13.7 \%$ $(P<0.05)$ and the latter was decreased by $23.9 \%(P<0.05)$ in the ala6-25Cys group. In the alarin + ala6-25Cys group, the RBP4 levels were elevated by $16.7 \%(P<0.05)$ but adiponectin contents were decreased by $23.5 \%(P<0.01)$ compared with the alarin group, while the former was decreased by $13.7 \%(P<0.05)$ but the latter was increased by $32.3 \%(P<0.05)$ when compared with the ala6-25Cys group. In addition, the former was elevated by $23.8 \%$ $(P<0.01)$ and the latter was reduced by $25.1 \%(P<0.01)$ in the diabetic control group compared with healthy controls.

\section{Glucose infusion rates}

The glucose infusion speed during the clamp tests was obviously increased by treatment with alarin as shown in Fig. $3(F(4,40)=25.4, P<0.001)$. When compared with diabetic controls, the infusion speed in the alarin group was increased by $22.9 \%(P<0.05)$, but in the ala6-25Cys group was decreased by $24.9 \%(P<0.05)$ (Fig. 3$)$. In the alarin +ala6-25Cys group, the speed was decreased by $18.7 \%(P<0.05)$ compared with the alarin group, but was elevated by $33.3 \% \quad(P<0.05)$ compared with the ala6-25Cys group. Similarly, compared with the healthy controls, the infusion rates were significantly reduced by $29.7 \%(P<0.01)$ in the diabetic control group.

\section{2-NBDG and Glut4 mRNA levels}

The central injection of alarin for 7 days vs vehicle significantly stimulated 2-NBDG uptake and Glut4 mRNA expression in adipocytes of T2DR $(F(4,40)=15.0$, $P<0.0001 ; F(4,40)=16.9, P<0.0001)$. Compared with the diabetic controls, the 2-NBDG and Glut4 mRNA expression levels were increased by $19.5 \%(P<0.05)$ and $33.1 \%(P<0.01)$ in the alarin group, and decreased by $12.8 \%(P<0.05)$ and $14.8 \%(P<0.05)$ in the alarin 6-25Cys group respectively (Fig. 4 ). Moreover, both parameters in the alarin+alarin 6-25Cys group in comparison to the alarin group were, respectively, attenuated by $12.6 \%$ $(P<0.05)$ and $20.7 \%(P<0.05)$, whereas in comparison to the alarin 6-25Cys group increased by $19.5 \%(P<0.05)$ and $21.4 \%(P<0.05)$. Compared with healthy controls, both

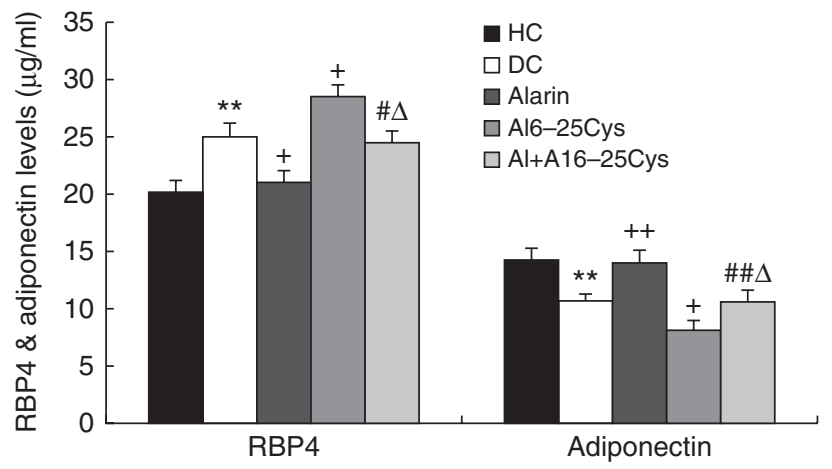

\section{Figure 2}

The changes in plasma RBP4 and adiponectin concentrations after the i.c.v. injection of alarin in type 2 diabetic rats $(n=8)$. The plasma RBP4 content was lower, but the adiponectin concentration was higher in alarin group compared with diabetic controls (DCs), which may be abolished by the alarin antagonist, alarin 6-25Cys. The former was higher, but the latter was lower in DCs compared with healthy controls (NCs) and in the alarin 6-25Cys group compared with DCs. While the former was lower, the latter was higher in the alarin + alarin 6-25Cys group compared with the alarin 6-25Cys group. All data shown are the means \pm S.E.M. ${ }^{*} P<0.01$ vs NCs; ${ }^{+} P<0.05,{ }^{++} P<0.01$ vs DCs; ${ }^{\#} P<0.05,{ }^{\# \#} P<0.01$ vs alarin group; ${ }^{\Delta} P<0.05$ vs alarin 6-25Cys group.

Published by Bioscientifica Ltd. 


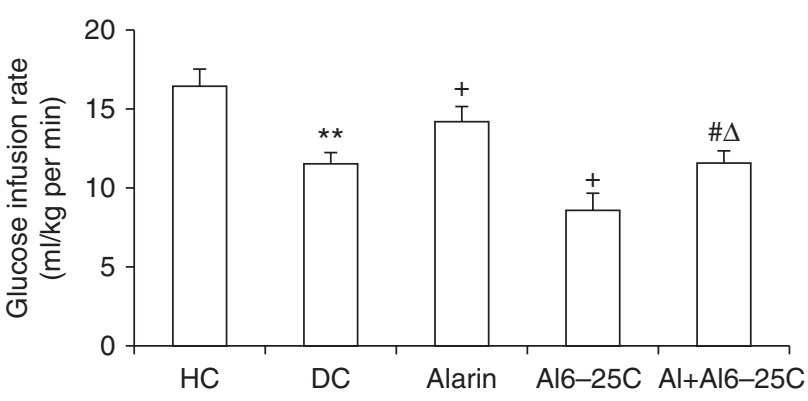

Figure 3

The central administration of alarin significantly elevated glucose infusion rates in the hyperinsulinemic-euglycemic clamp tests in type 2 diabetic rats $(n=8)$. The infusion rates were increased in the alarin group compared with the diabetic control (DC, $n=8$ ), and in the alarin + alarin 6-25Cys group (Al+Al6-25C) compared with the alarin 6-25Cys group (Al6-25C). Moreover, the infusion rates in alarin + alarin 6-25Cys group and alarin 6-25Cys group were lower than those in the alarin group and DCs respectively. The rates were lower in DCs compared with healthy controls (NCs). The data shown are the means \pm s.E.M. ${ }^{* *} P<0.01$ vs NCs; ${ }^{+} P<0.05$ vs DCs; ${ }^{\#} P<0.05$ vs alarin group; ${ }^{\Delta} P<0.05$ vs alarin $6-25$ Cys group.

indexes in the diabetic control group were reduced by $16.2 \%(P<0.05)$ and $25.4 \%(P<0.01)$ respectively.

\section{GLUT4 contents in membranes of adipocytes}

We used the western blot assay to evaluate the effects of central alarin on the GLUT4 expression levels in adipocytes. The i.c.v. treatment with alarin significantly elevated GLUT4 protein levels in plasma membranes $(F(4,40)=76.9, P<0.0001)$ and total cell plasma membranes $(F(4,40)=61.2, P<0.0001)$ of adipocytes as shown in Fig. 5. Compared with the diabetic controls, the GLUT4 immunoreactivities in the alarin group were elevated by $128.1 \%(P<0.01)$ in plasma membranes and $11.3 \%$ $(P<0.05)$ in total cell membranes, while in the ala625Cys group were reduced by $18.3 \%(P<0.05)$ in plasma membranes and $8.9 \%(P<0.05)$ in total cell membranes. The immunoreactivities in the alarin +alarin 6-25Cys group were decreased by $35.5 \%(P<0.01)$ in plasma membranes and $10.2 \%(P<0.05)$ in total cell membranes in comparison to the alarin group, but were elevated by $66.6 \%(P<0.01)$ in plasma membranes and $11.3 \%$ $(P<0.05)$ in total cell membranes in comparison to the ala6-25Cys group. In addition, the GLUT4 protein levels in plasma membranes and total cell membranes of the diabetic control group were reduced by $36.7 \%(P<0.01)$ and $23.3 \%(P<0.01)$ compared with each healthy control. The ratios of GLUT4 densities in plasma membranes to total cell membranes were $14.4,11.9,24.5,10.8$, and
$19.1 \%$ in healthy control, diabetic control, alarin, ala625Cys, and alarin + ala6-25Cys groups respectively.

\section{VAMP2 concentration in plasma membranes}

We measured the VAMP2 levels in plasma membranes of adipocytes using subcellular fractionation and western blotting assay. The VAMP2 levels in plasma membranes of adipocytes were significantly elevated by central injection of alarin $(F(4,40)=26.9, P<0.0001)$. In comparison to the diabetic controls, VAMP2 levels in the alarin group were increased by $28.4 \%(P<0.05)$, and in the alarin $6-25$ Cys group reduced by $29.7 \%(P<0.05)$ (Fig. 6$)$. The VAMP2 contents in the alarin + alarin 6-25Cys group were reduced by $24.4 \%(P<0.05)$ compared with the alarin group and elevated by $38.9 \%(P<0.05)$ compared with the alarin 6-25Cys group respectively. Again, the VAMP2 levels in plasma membranes in the diabetic control group were decreased by $35.7 \%(P<0.01)$ compared with healthy controls.

\section{Discussion}

Obesity is a prevalent public health problem and a major risk factor for the development of insulin resistance, fatty liver, and T2DR. Several studies have clearly established that injection of alarin directly into the paraventricular nucleus significantly increased daily caloric intake and

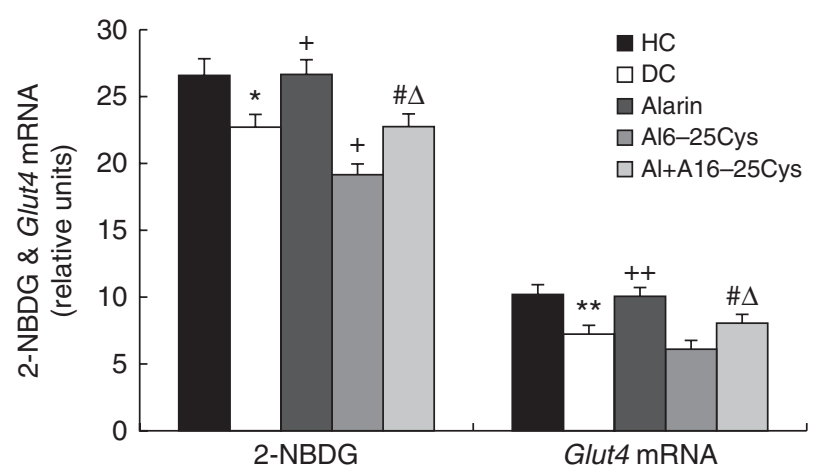

\section{Figure 4}

The i.c.v. administration of alarin significantly upregulated 2-NBDG and Glut4 mRNA expression levels in adipocytes $(n=8)$. The 2-NBDG and Glut4 mRNA levels after central injection of alarin were increased compared with the diabetic control ( $D C, n=8$ ). Both the indexes in the alarin + alarin 6-25Cys group were lower than those in the alarin group, but higher than those in the alarin 6-25Cys group. Moreover, both indexes were lower in DCs compared with healthy controls (NCS) and in the alarin 6-25Cys group compared with DCs. The data shown are the means \pm S.E.M. ${ }^{*} P<0.05$,

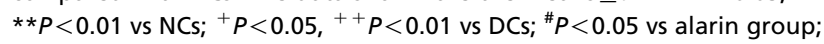
${ }^{\Delta} P<0.05$ vs alarin $6-25$ Cys group. 


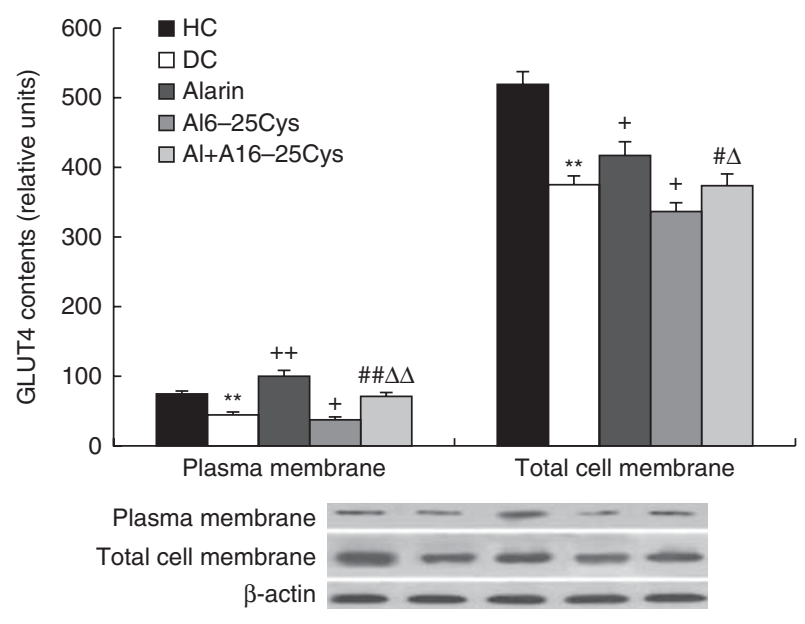

\section{Figure 5}

The i.c.v. injection of alarin upregulated GLUT4 expression and trafficking in adipocytes $(n=8)$. The central treatment with alarin elevated GLUT4 immunoreactivity in plasma membranes and total cell membranes of adipocytes compared with the diabetic controls (DCs). Moreover, the immunoreactivity in both the membranes of adipocytes was lower in the alarin + alarin 6-25Cys group than in the alarin group, but higher when compared with the alarin 6-25Cys group. In addition, the immunoreactivity was lower in DCs compared with healthy controls (NCs) and in the alarin 6-25Cys group compared with DCs. The sum of the GLUT4 concentration in plasma membranes and that in intracellular membranes was calculated as the GLUT4 concentration of total cell membranes. The data shown are the means \pm s.E.M. ${ }^{*} P<<0.01$ vs NCs; ${ }^{+} P<0.05,{ }^{++} P<0.01$ vs DCs; ${ }^{*} P<0.05$, ${ }^{\# \#} P<0.01$ vs alarin group; ${ }^{\Delta} P<0.05,{ }^{\Delta \Delta} P<0.01$ vs alarin $6-25$ Cys group. The sequence of a series of representative western blots lines in each panel is $\beta$-actin, NCs, DCs, alarin group, alarin 6-25Cys group, and alarin +alarin 6-25Cys group.

body weight of animals (Boughton et al. 2010, Van Der Kolk et al. 2010, Fraley et al. 2012). The alarin-induced gain of body weight in mice could be due to not only an increase in food and water intake, but also a decrease in excretion of feces and urine, and metabolic rate (Fraley et al. 2012). Consistent with these results, the current study provided further evidences that central injection of alarin, such as galanin and GALP, may increase the body weight of animals. However, whether or not central injection of alarin could increase food intake of rats in fasted or early dark-phase experimental paradigms is yet to be elucidated (Boughton et al. 2010). The orexigenic effect of alarin appears to be relatively weak when compared with other hypothalamic orexigenic neuropeptides such as neuropeptide Y (NPY; Levine \& Morley 1984). Alarin did stimulate NPY release from hypothalamic explants (Boughton et al. 2010). Therefore, the orexigenic effect of alarin is at least partly mediated by the increase in hypothalamic NPY release. To date, we have no idea as to which regions of the brain mediate the effect of alarin on the regulation of food intake and body weight. A study with the anti-alarin antibody revealed alarin-like immunoreactivity in the locus coeruleus and subcoeruleus (Eberhard et al. 2012). Using anterograde tracing, the projections from the hypothalamus to the locus coeruleus have been found (Van Der Kolk et al. 2010). These areas of the brain are implicated in the regulation of appetite and food intake (Boughton et al. 2010). Thus, it is possible that a central alarin-induced increase in feeding and body weight may be achieved through these structures. Furthermore, more studies are required to explore the precise region of the brain involved in the orexigenic role of alarin.

A large amount of data indicated that increased circulating adipokine levels, including RBP4 and adiponectin, may be used as biomarkers to assess the development of insulin resistance (Fang et al. 2014). RBP4, produced in the liver and mature lipid-accumulating adipocytes, is a lypocalin protein transporting retinol in the circulation (Yamaaki et al. 2013). Moreover, adiponectin is a potent insulin-sensitizing and anti-inflammatory hormone (Patel et al. 2012). The enhanced RBP4 and reduced adiponectin levels are correlated with obesity, insulin resistance, and the development of early T2DR (Kotnik et al. 2013, Meyer et al. 2013, Yoon et al. 2013). In order to determine the effect of central alarin on RBP4 and adiponectin liberation, we contrasted the plasma

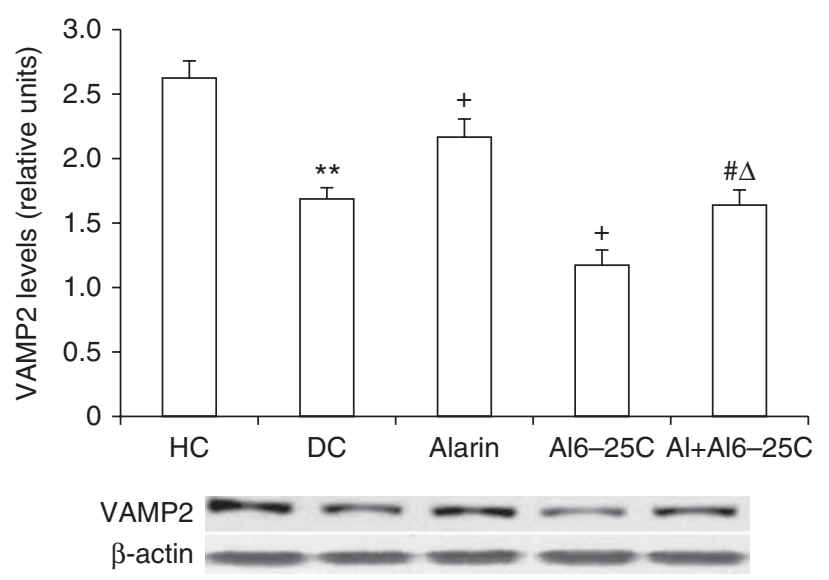

Figure 6

The i.c.v. administration of alarin significantly elevated the VAMP2 levels in plasma membranes of adipocytes $(n=8)$. The VAMP2 contents were increased in the alarin group compared with the diabetic control (DC, $n=8$ ), and in the alarin + alarin 6-25Cys group (Al+Al6-25C) compared with the alarin 6-25Cys group (Al6-25C). The contents in alarin + alarin $6-25$ Cys and alarin 6-25Cys groups were lower than those in the alarin group and DCs respectively. The levels were lower in DCs than healthy controls (NCS). The data shown are the means \pm S.E.M. $* * P<0.01$ vs NCs; ${ }^{+} P<0.05$ vs $D C s ;{ }^{\#} P<0.05$ vs alarin group; ${ }^{\Delta} P<0.05$ vs alarin $6-25$ Cys group. 
concentration in the alarin group with the diabetic controls. The results indicated that central administration of alarin significantly decreased RBP4 levels, but increased adiponectin levels in adipocytes, which may be attenuated by pretreatment with alarin 6-25Cys, suggesting that the alarin projective system may inhibit RBP4 but increase adiponectin secretion to ameliorate insulin resistance via activating central alarin receptors in T2DR rats. As alarin does not share any homology with galanin, it is impossible for alarin to compete with galanin for known galanin receptors, GalR1-3 (Van Der Kolk et al. 2010). The central effects of alarin on insulin resistance seem to be mediated by unknown specific alarin receptors in the brain. As well, it is possible that there may be some unidentified galanin receptor(s) that can also be activated by alarin.

2-NBDG, a newly developed fluorescent 2-deoxyglucose analog, may be transported into cells via the same GLUT as glucose (Sheth et al. 2009). After being absorbed into cells, 2-NBDG is phosphorylated to 2-NBDG-6-P, which prevents it from being released again from the cells and metabolized further as it lacks the necessary 2-hydroxyl group for glycolysis. Therefore, these features made the distribution of trapped 2-NBDG through glucose transporters a good reflection of cellular glucose uptake and an assessment of insulin resistance $\left(\mathrm{O}^{\prime} \mathrm{Neil}\right.$ et al. 2005). It is known that central galanin is involved in the regulation of glucose intake and energy balance (Zhang et al. 2012). In the same way, i.c.v. treatment with alarin elevated 2-NBDG contents, which were abrogated by pretreatment with alarin 6-25Cys in the current study, i.e. the central alarin projective system may reduce blood glucose and increase glucose uptake in adipocytes of T2DR. As mentioned earlier, alarin can stimulate pleiotropic NPY release from hypothalamic explants. NPY may directly enter into the adipose tissue via activation of NPY-Y2 receptors to accelerate glucose intake, resulting in stimulating appetite and obesity (Kuo et al. 2008). Central alarin-induced increase in glucose uptake and reduction in blood glucose, observed in the present study, are also part of the roles played by the NPY.

The hyperinsulinemic-euglycemic clamp is a 'gold standard' for assessment of insulin sensitivity and peripheral glucose disposal rate (Catalogna et al. 2012). During the clamp test, a constant insulin infusion facilitates the circulating glucose uptake into insulin-sensitive tissues and inhibits endogenous glucose production by the liver. The decline in the plasma glucose level induced by insulin infusion is prevented by an exogenous glucose perfusion in a concomitant variable rate. Then, the amount of exogenous glucose, required to maintain plasma glucose at its clamp level, is quantified by the glucose infusion rate that presents insulin sensitivity in the subject. An elevation in the glucose infusion speed suggests an increase in insulin sensitivity. In the current study, the glucose infusion rates in the alarin group were higher than those in diabetic controls in the clamp tests, which were antagonized by pretreatment with alarin 6-25Cys, suggesting that the equilibrium point of glucose production vs glucose utilization was shifted toward benefiting the latter by the central injection of alarin. Glucose disposal is enhanced after administration of alarin, resulting in an increase in insulin sensitivity and a decrease in the plasma glucose level in the animals.

A number of studies have demonstrated that the Glut4 mRNA level reflects a change in Glut4 mRNA synthesis rather than its half-life (Liang et al. 2012). The nuclear runon assays indicated that the transcription of Glut4 was decreased in streptozotocin-induced diabetic animals. The streptozotocin-dependent changes in the Glut4 mRNA expression occur much more rapidly in adipose tissue than in skeletal muscle. In line with these, this study demonstrated that the central treatment with alarin upregulated Glut4 mRNA expression levels, which was attenuated by pretreatment with alarin 6-25Cys in the adipocytes of T2DR rats, suggesting that the central infusion of alarin is effective to increase GLUT4 synthesis in adipose cells as central galanin does (Zhang et al. 2012). Interestingly, i.c.v. injection of alarin increased c-Fos activity in the nucleus tractus solitarius and other centers of the hindbrain (Van Der Kolk et al. 2010), suggesting that alarin in the brain may affect much of the central transmitter release to influence Glut4 mRNA expression in adipocytes of subjects.

The densitometry of adipocytes revealed that the central administration of alarin significantly increases GLUT4 contents in both plasma membranes and total cell membranes of adipocytes compared with diabetic controls. The ratios of GLUT4 contents in the former (24.5\%) to the latter (11.9\%) were higher in the alarin group than in diabetic controls, which may be abolished by alarin 6-25Cys (19.1\%), suggesting that the central injection of alarin may increase not only GLUT4 amounts, but also GLUT4 trafficking from cytoplasmic membranes to plasma membranes, similar to the action of galanin, to benefit glucose uptake and insulin sensitivity in adipocytes of T2DR (Liang et al. 2012).

GLUT4-containing vesicular trafficking between intracellular membrane compartments and plasma membranes involves the physical fusion of trafficking vesicles with the

Published by Bioscientifica Ltd 
target membrane compartment. VAMP2, a member of the vesicle-associated membrane protein family, is essential to catalyze GLUT4 vesicle docking and fusing with plasma membranes of cells (Williams \& Pessin 2008). The quantity of VAMP2-positive GLUT4 storage vesicles in plasma membranes represents the specialized GLUT4 storage pool that may be rapidly exocytosed in response to stimuli. An injection of VAMP2 neurotoxins or the overexpression of dominant interfering VAMP2 peptides inhibited GLUT4 translocation. The current study found that central administration of alarin significantly enhanced AMP2 concentration in the plasma membranes, which may be inhibited by pretreatment with alarin 6-25Cys. This suggests that alarin-induced GLUT4 trafficking is triggered by enhanced VAMP2 levels in plasma membranes. Notably, i.c.v. injection of alarin significantly increased the release of luteinizing hormone and gonadotropin-releasing hormone from the hypothalamus, which was blocked by pretreatment with the GnRH antagonist cetrorelix in rats (Boughton et al. 2010, Van Der Kolk et al. 2010), indicating that central alarin regulates the activity of the hypothalamic-pituitary-target gland axis in rodents. Accordingly, it may be speculated that central alarin subserves VAMP2 and GLUT4 translocation possibly via regulating the activity of hypothalamic-pituitarytarget gland axis, which should been explored further.

In short, the current study results indicated that central administration of alarin increased body weight, adiponectin release, 2-NBDG uptake, Glut4 mRNA expression, glucose infusion rates in the hyperinsulinemiceuglycemic clamp test, and GLUT4 and VAMP2 translocation to cell surfaces, but reduced the blood glucose level and RBP4 discharge in the T2DR rat. These findings suggest that the alarin-VAMP2-GLUT4 pathway is involved in the beneficial effect of central alarin on glucose uptake and insulin sensitivity in adipocytes of T2DR animals. This study contributes to our understanding of the antidiabetic role of the central alarin projective system and suggests that the alarin receptor could be a potential therapeutic target for T2DR.

\section{Declaration of interest}

The authors declare that there is no conflict of interest that could be perceived as prejudicing the impartiality of the research reported.

\section{Funding}

This work was supported by grants from the National Natural Scientific Fund of China (81173392) and the National Health and Family Planning Commission of China (W201309).

\section{References}

Absalan A, Mohiti-Ardakani J, Hadinedoushan H \& Khalili MA 2012 Hydroalcoholic cinnamon extract, enhances glucose transporter isotype- 4 translocation from intracellular compartments into the cytoplasmic membrane of C2C12 myotubes. Indian Journal of Clinical Biochemistry 27 351-356. (doi:10.1007/s12291-012-0214-y)

Boughton CK, Patterson M, Bewick GA, Tadross JA, Gardiner JV, Beale KE, Chaudery F, Hunter G, Busbridge M, Leavy EM et al. 2010 Alarin stimulates food intake and gonadotrophin release in male rats. British Journal of Pharmacology 161 601-613. (doi:10.1111/j.1476-5381.2010. 00893.x)

Bu L, Liu Z, Zou J, Gao X, Bao Y \& Qu S 2013 Blocking central galanin receptors attenuates insulin sensitivity in myocytes of diabetic trained rats. Journal of Neuroscience Research 91 971-977. (doi:10.1002/jnr. 23228)

Catalogna M, Cohen E, Fishman S, Halpern Z, Ne vo U \& Ben-Jacob E 2012 Artificial neural networks based controller for glucose monitoring during clamp test. PLoS ONE 7 e44587-446597. (doi:10.1371/journal. pone.0044587)

Eberhard N, Mayer C, Santic R, Navio RP, Wagner A \& Bauer HC 2012 Distribution of alarin immunoreactivity in the mouse brain. Journal of Molecular Neuroscience 46 18-32. (doi:10.1007/s12031-011-9546-y)

Fang PH, Shi MY, Yu M, Guo LL, Bo P \& Zhang ZW 2014 Endogenous peptides as risk markers to assess the development of insulin resistance. Peptides 51 9-14. (doi:10.1016/j.peptides.2013.10.025)

Fraley GS, Leathley E, Lundy N, Chheng E, King I \& Kofler B 2012 Effects of alarin on food intake, body weight and luteinizing hormone secretion in male mice. Neuropeptides 46 99-104. (doi:10.1016/j.npep.2011.12.003)

Fraley GS, Leathley E, Nickols A, Gerometta E, Coombs E, Colton S, Gallemore S, Lindberg A \& Kofler B 2013 Alarin 6-25Cys antagonizes alarin-specific effects on food intake and luteinizing hormone secretion. Neuropeptides 47 37-41. (doi:10.1016/j.npep.2012. 08.007)

Guo L, Shi M, Zhang L, Li G, Zhang LH, Shao H, Fang P, Ma Y, Li J, Shi Q et al. 2012 Galanin antagonist increases insulin resistance by reducing glucose transporter 4 effect in adipocytes of rats. General and Comparative Endocrinology 173 159-163. (doi:10.1016/j.ygcen.2011.05.011)

Kotnik P, Keuper M, Wabitsch M \& Fischer-Posovszky P 2013 Interleukin$1 \beta$ downregulates RBP4 secretion in human adipocytes. PLOS ONE 8 e57796-e57805. (doi:10.1371/journal.pone.0057796)

Kuo LE, Czarnecka M, Kitlinska JB, Tilan JU, Kvetnanský R \& Zukowska Z 2008 Chronic stress, combined with a high-fat/high-sugar diet, shifts sympathetic signaling toward neuropeptide $\mathrm{Y}$ and leads to obesity and the metabolic syndrome. Annals of the New York Academy of Sciences 1148 232-237. (doi:10.1196/annals.1410.035)

Levine AS \& Morley JE 1984 Neuropeptide Y: a potent inducer of consummatory behavior in rats. Peptides 5 1025-1029. (doi:10.1016/ 0196-9781(84)90165-7)

Liang Y, Sheng S, Fang P, Ma YP, Li J, Shi QJ, Sui Y \& Shi M 2012 Exerciseinduced galanin release facilitated GLUT4 translocation in adipocytes of type 2 diabetic rats. Pharmacology, Biochemistry, and Behavior 100 554-559. (doi:10.1016/j.pbb.2011.10.026)

Meyer LK, Ciaraldi TP, Henry RR, Wittgrove AC \& Phillips SA 2013 Adipose tissue depot and cell size dependency of adiponectin synthesis and secretion in human obesity. Adipocyte 2 217-226. (doi:10.4161/adip. 24953)

O'Neil RG, Wu L \& Mullani N 2005 Uptake of a fluorescent deoxyglucose analog (2-NBDG) in tumor cells. Molecular Imaging and Biology 7 388-392. (doi:10.1007/s11307-005-0011-6)

Patel SA, Hoehn KL, Lawrence RT, Sawbridge L, Talbot NA, Tomsig JL, Turner N, Cooney GJ, Whitehead JP, Kraegen EW et al. 2012 Overexpression of the adiponectin receptor AdipoR1 in rat skeletal muscle amplifies local insulin sensitivity. Endocrinology 153 5231-5246. (doi:10.1210/en.2012-1368)

Published by Bioscientifica Ltd. 
Santic R, Fenninger K, Graf K, Schneider R, Hauser-Kronberger C, Schilling FH, Kogner P, Ratschek M, Jones N, Sperl W et al. 2006 Gangliocytes in neuroblastic tumors express alarin, a novel peptide derived by differential splicing of the galanin-like peptide gene. Journal of Molecular Neuroscience 29 145-152. (doi:10.1385/JMN:29:2:145)

Santic R, Schmidhuber SM, Lang R, Rauch I, Voglas E, Eberhard N, Bauer JW, Brain SD \& Kofler B 2007 Alarin is a vasoactive peptide. PNAS 12 1021710222. (doi:10.1073/pnas.0608585104)

Sheth RA, Josephson L \& Mahmood U 2009 Evaluation and clinically relevant applications of a fluorescent imaging analog to fluorodeoxyglucose positron emission tomography. Journal of Biomedical Optics $\mathbf{1 4}$ 064014-064027. (doi:10.1117/1.3259364)

Song X, Lichti CF, Townsend RR \& Mueckler M 2013 Single point mutations result in the miss-sorting of Glut4 to a novel membrane compartment associated with stress granule proteins. PLOS ONE 8 e68516-e68531. (doi:10.1371/journal.pone.0068516)

Van Der Kolk N, Madison FN, Mohr M, Eberhard N, Kofler B \& Fraley GS 2010 Alarin stimulates food intake in male rats and LH secretion in castrated male rats. Neuropeptides 44 333-340. (doi:10.1016/j.npep.2010.04.001)

Wada A, Wong PF, Hojo H, Hasegawa M, Ichinose A, Llanes R, Kubo Y, Senba M \& Ichinose Y 2013 Alarin but not its alternative-splicing form,
GALP (galanin-like peptide) has antimicrobial activity. Biochemical and Biophysical Research Communications 434 223-227. (doi:10.1016/ j.bbrc.2013.03.045)

Williams D \& Pessin JE 2008 Mapping of R-SNARE function at distinct intracellular GLUT4 trafficking steps in adipocytes. Journal of Cell Biology 180 375-387. (doi:10.1083/jcb.200709108)

Yamaaki N, Yagi K, Kobayashi J, Nohara A, Ito N, Asano A, Nakano K, Liu J, Okamoto T, Mori Y et al. 2013 Impact of serum retinol-binding protein 4 levels on regulation of remnant-like particles triglyceride in type 2 diabetes mellitus. Journal of Diabetes Research 2013 143515-143522. (doi:10.1155/2013/143515)

Yoon JH, Kim SK, Choi HJ, Choi SI, Cha SY, Koh SB, Kang HT \& Ahn SV 2013 Adiponectin provides additional information to conventional cardiovascular risk factors for assessing the risk of atherosclerosis in both genders. PLoS ONE 8 e75535-e75541. (doi:10.1371/journal.pone. 0075535)

Zhang Z, Sheng S, Guo L, Li G, Zhang L, Zhang L, Shi M, Bo P \& Zhu Y 2012 Intracerebroventricular administration of galanin antagonist sustains insulin resistance in adipocytes of type 2 diabetic trained rats. Molecular and Cellular Endocrinology 361 213-218. (doi:10.1016/j. mce.2012.04.012)

Received in final form 3 September 2014

Accepted 18 September 2014

Accepted Preprint published online 18 September 2014
(C) 2014 Society for Endocrinology Printed in Great Britain
Published by Bioscientifica Ltd. 